\title{
Revisiting the minimum set cover, the maximal coverage problems and a maximum benefit area selection problem to make climate-change-concerned conservation plans effective
}

\author{
Diogo Alagador $^{1}$ (D) | Jorge Orestes Cerdeira ${ }^{2}$ (I)
}

\author{
${ }^{1}$ The "Rui Nabeiro" Biodiversity Chair, \\ MED-Mediterranean Institute for \\ Agriculture, Environment and Development, \\ Universidade de Évora, Évora, Portugal \\ ${ }^{2}$ Department of Mathematics and Centre for \\ Mathematics and Applications, Faculdade de \\ Ciências e Tecnologia, Universidade NOVA \\ de Lisboa, Costa da Caparica, Portugal

\section{Correspondence} \\ Diogo Alagador \\ Email: alagador@uevora.pt \\ Funding information \\ Fundação para a Ciência e a Tecnologia, \\ Grant/Award Number: PTDC/AAG- \\ GLO/3979/2014, SFRH/BPD/104077/2014 \\ and UID/MAT/00297/2019
}

Handling Editor: Robert Freckleton

\begin{abstract}
1. Informed decisions for the selection of protected areas (PAs) are grounded in two general problems in Operations Research: the minimum set covering problem (minCost), where a set of ecological constraints are established as conservation targets and the minimum cost PAs are found, and the maximal coverage problem (maxCoverage) where the constraint is uniquely economic (i.e. a fixed budget) and the goal is to maximize the number of species having conservation targets adequately covered.
\end{abstract}

2. We adjust minCost and maxCoverage to accommodate the dynamic effects of climate change on species' ranges. The selection of sites is replaced by the selection of time-ordered sequences of sites (climate change corridors), and an estimate of the persistence of each species in corridors is calculated according to the expected suitability of each site in the respective time period and the capacity of species to disperse between consecutive sites along corridors. In these problems, conservation targets are expressed as desired (and attainable) species persistence levels. We also introduce a novel problem (minShortfall) that combines minCost and maxCoverage. Unlike these two problems, minShortfall allows persistence targets to be missed and minimizes the sum of those gaps (i.e. target shortfalls), subject to a limited budget.

3. We illustrate the three problems with a case study using climatic suitability estimates for 10 mammal species in the Iberian Peninsula under a climate change scenario until 2080. We compare solutions of the three problems with respect to species persistence and PA costs, under distinct settings of persistence targets, number of target-fulfilled species and budgets. The solutions from different problems differed with regard to the areas to prioritize, their timings and the species whose persistence targets were fulfilled. This analysis also allowed identifying groups of species sharing corridors in optimal solutions, thus allowing important financial savings in site protection.

4. We suggest that enhancing species persistence is an adequate approach to cope with habitat shifts due to climate change. We trust the three problems discussed can provide complementary and valuable support for planners to anticipate decisions in order that the negative effects of climate change on species' persistence are minimized. 


\section{KEYWORDS}

climate change, connectivity, conservation plan, conservation targets, decision support, optimization, persistence, reserve design

\section{1 | INTRODUCTION}

Effective biodiversity conservation strongly relies on the establishment and management of protected areas (PAs; Watson, Dudley, Segan, \& Hockings, 2014). In a world pervasively dominated by the economic uses of land, PAs are costly to establish and often conflict with dominant socio-economic activities (Margules \& Pressey, 2000). In recent decades, a large number of studies have proposed optimized approaches for PA selection aiming to maximize conservation benefits at minimum cost (reviewed in McIntosh, Pressey, Lloyd, Smith, \& Grenyer, 2017; Moilanen, Wilson, \& Possingham, 2009). These approaches are grounded in two general (and related) PA selection problems. In the minimum set covering problem (minCost), a conservation target (often a representation target; e.g. total area, or number of sites, or allele frequency) is defined for each species with the objective of identifying a set of PAs of minimum cost, in which all the established conservation targets (hereafter targets) are met (Saetersdal, Line, \& Birks, 1993). The maximal coverage problem (maxCoverage) maximizes the number of species adequately represented in PAs, when financial or areal resources are limited (Church, Stoms, \& Davis, 1996). In its simplest form, maxCoverage assumes a species is adequately protected when its representation in final solutions are limited to a single spatial unit (a site, hereafter). However a generalized, precautionary version of the problem exists, in which a species is considered adequately protected if it occurs in multiple sites in the final solution (Camm, Norman, Polasky, \& Solow, 2002; Snyder \& Haight, 2014). Well-known area prioritization softwares-Marxan (Ball, Possingham, \& Watts, 2009) and Zonation (Moilanen, Kujala, \& Leathwick, 2009)-incorporate algorithms to search for approximations to optimal solutions for both the minCost and maxCoverage problems.

In the last two decades, climate change has emerged as a significant threat to biodiversity (McLaughlin, Hellmann, Boggs, \& Ehrlich, 2002; Thomas et al., 2004; Walther et al., 2002). Therefore, the integration of climate dynamics in conservation planning is an urgent, priority task (Jones, Watson, Possingham, \& Klein, 2016). The effectiveness of static PAs in covering important habitats of threatened species is particularly vulnerable to the adaptive redistribution of species ranges as their suitable climates shifts spatially. Multiple studies have addressed how PAs will perform (or are performing) under different scenarios of climate change (Araújo, Alagador, Cabeza, NoguésBravo, \& Thuiller, 2011; Bagchi et al., 2013; Baker et al., 2015; D'Amen et al., 2011; Rutherford, Powrie, \& Schulze, 1999; Soto, 2001). Other studies advanced new frameworks to optimize PA location in order that adequate species protection levels are preserved over time as climate changes (Albert Cécile, Rayfield, Dumitru, \& Gonzalez, 2017; Araújo, 2009; Beier, 2012; D'Aloia et al., 2019; Watson et al., 2011). One line of study, introduced by Williams et al. (2005) and followed by
Phillips, Williams, Midgley, and Aaron (2008) and Alagador, Cerdeira, and Araújo (2014), uses the paradigm of dispersal corridors (herein climate-change corridors, CCCs) for the selection of PAs. A CCC defines a sequence of sites, each one representing a time period. Conceptually, a CCC defines a spatial trajectory used by a species' population over time. Alagador et al. (2014) advanced a measure to assess the persistence of a species in a CCC which, as in Levin's model for metapopulation dynamics (Ovaskainen \& Hanski, 2003a), incorporates two distinct ecological processes: environmental suitability and dispersal/settlement success. The persistence of a species in a set of sites is estimated by the product of environmental suitability and dispersal success over the ordered sequence of CCC sites.

Contrary to representation targets (based on minimum number of occurrences or abundance data), persistence targets do not have a straightforward spatial interpretation (Alagador \& Cerdeira, 2017; Di Marco et al., 2016; Keith et al., 2014). They may be seen as a sequence of representational (environmentally suitable) targets that move over time (accounting to species-specific dispersal ability). Setting persistence targets is more complex than finding adequate representation targets, since changes of environmental suitability over time are considered together with species-specific dispersal patterns. To define accurate persistence targets, it may be convenient to produce, for each species separately, solutions with varying target values and then set the final target from the analysis of the different solutions obtained.

Here, we re-formulate and re-interpret the minCost and the maxCoverage to guide the selection of PAs across time, under climate change, using CCCs and explicit persistence goals. We also introduce a novel third problem (minShortfall) which, unlike the previous ones, accounts for the benefits of protecting corridors for a species even when, those corridors fail to meet the persistence target (i.e. result in a target shortfall). An optimal solution of minShortfall is a set of CCCs that minimizes the sum of species shortfalls, subject to limited budget or area.

We use a dataset that illustrates the predicted climatic suitability for 10 mammal species of conservation concern in the Iberian Peninsula (Iberia for short) from the baseline period until 2080, to compare solutions of the three problems regarding species persistence and PA costs, under varying values of persistence targets, number of target-fulfilled species and budgets. We identify the species whose protection cost (i.e. the cost associated with their selected CCCs) most limits the persistence of the remaining species in the PAs. We also estimate how much the persistence performance of the optimized sets of CCCs, identified for each single species without budgets limitations, deteriorates when compared to persistence values in solutions for multiple species with varying budget limitations. This analysis allows detection of the species that most 
limit the adequate protection of other species and identification of groups of species that share the same CCCs.

\section{2 | MATERIALS AND METHODS}

\section{1 | Climate change corridors}

We modelled the selection of PAs to accommodate the dynamic effects of climate change on species' ranges (Alagador, Cerdeira, \& Araújo, 2016). Under this framework, the selectable planning-units are CCCs. A CCC is a time-ordered sequence of (possibly not distinct) sites, each one assigned to a period of time (i.e. sites $I=\left(i_{0}, i_{1}, \ldots\right.$, $\left.i_{N-1}, i_{N}\right)$ assigned to time periods $T=\left(t_{0}, t_{1}, t_{N-1}, t_{N}\right)$ respectively; see Table 1 for a description of the mathematical nomenclature).

A measure of persistence for each species in each CCC may be estimated combining two factors: (a) the probability (or a comparable zero-to-one score) of a site $i$ to be suitable for species $s$ to occur (i.e. viable populations) in a given time period, $t,\left(o_{s}^{i t}\right)$ and $(b)$ the probability (or a comparable zero-to-one score) of species $s$ to disperse successfully from one site, $i$, to another site, $j$, in a given period of time $\left(d_{s}^{i j}\right)$.

TABLE 1 Variables and parameters used in the formulations of the CCC selection problems

\begin{tabular}{|c|c|}
\hline Symbol & Description \\
\hline$S$ & Set of species \\
\hline 1 & Set of sites \\
\hline$T$ & Set of time periods \\
\hline$s$ & Index for a species \\
\hline$i$ & Index for a site \\
\hline$t$ & Index for a time period \\
\hline C & Set of corridors available for selection \\
\hline$C^{i t}$ & $\begin{array}{l}\text { Set of corridors from } C \text { that intersect at site } i \text { at time } \\
\text { period } t\end{array}$ \\
\hline c & Index for a corridor \\
\hline$o_{s}^{i t}$ & $\begin{array}{l}\text { Probability/suitability of site } i \text { for the occurrence of } \\
\text { species } s \text { and time period } t\end{array}$ \\
\hline$d_{s}^{i j}$ & $\begin{array}{l}\text { Probability/likelihood of species } s \text { to disperse } \\
\text { successfully from site } i \text { to } j \text { in a given time-interval }\end{array}$ \\
\hline$P_{s}^{c}$ & Persistence of species $s$ in $\mathrm{CCC} c$ \\
\hline Cost $^{i t}$ & Cost (or area) of site $i$ at time period $t$ \\
\hline$t_{s}$ & The target defined for species $s$ \\
\hline K & Number of species to cover \\
\hline$B^{t}$ & Budget available for time period $t$ \\
\hline$z_{s}^{c}$ & $\begin{array}{l}\text { Variable to identify if CCC } c \text { of species } s \text { is to be selected } \\
\text { (1) or not (0) }\end{array}$ \\
\hline$x^{i t}$ & $\begin{array}{l}\text { Variable to identify if site } i \text { is to be selected (1) or not (0) } \\
\text { at time period } t\end{array}$ \\
\hline$\Delta_{s}$ & A variable to measure the gap of species s to its target $t_{s}$ \\
\hline$Y_{s}$ & $\begin{array}{l}\text { An auxiliary variable that informs if species } s \text { is } \\
\text { adequately represented in final solutions (1), or not (0) }\end{array}$ \\
\hline
\end{tabular}

We used the measure of persistence, $P_{s}^{c}$, of a species $s$ along a CCC, $c$, introduced in Alagador et al. (2014). There, $P_{s}^{c}$ is defined as the product of (a) the occurrence probabilities (or local suitability) of sites in CCC, $c$, at each of $N$ time periods and (b) the probabilities (or other success index) to move between sites in CCC with each time interval:

$$
P_{s}^{c}=o_{s}^{i_{0} t_{0}} \times d_{s}^{i_{i} i_{1}} \times o_{s}^{i_{1} t_{1}} \times \cdots \times o_{s}^{i_{N-1} t_{N-1}} \times d_{s}^{i_{N-1} i_{N}} \times o_{s}^{i_{N} t_{N}} .
$$

This product of probabilities reflects the probability of all the factorial events to occur (in this case, the probability of a species being part of a chain of sites and to disperse within that chain). In order to select CCCs that are robust to negative contagious effects originating from the spread of epidemics, founder effects, etc., the persistence of a species in final solutions is only evaluated in a set of spatially independent CCCs. Two CCCs are independent if they do not include the same site in the same time period. We defined the persistence of a species in a set $(C)$ of CCCs to be the maximum sum of the persistence scores in a subset $\left(C^{\prime}\right)$ of independent CCCs of $C$. For computational tractability, it may be reasonable, instead of considering all CCCs for each species, to restrict the analysis to a pool of $n_{s}$ pre-selected CCCs that present the largest persistence scores for that species. This is not a serious limitation because, for each species, most CCCs will have zero or near-zero persistence score, and so they are unlikely to be selected in solutions. The candidate set of $n_{s}$ CCCs can be easily generated using a k-shortest path algorithm (e.g. Fox, 1975; Martins, Pascoal, \& Santos, 1999) which, in a directed graph with non-negative edge weights, two vertices $u$ and $v$, and a positive integer $k$, delivers the $k$ minimum cost paths from $u$ to $v$ in increasing order of cost. For acyclic graphs, which is the case of our application, these algorithms are extremely fast.

\section{2 | Planning designs for multiple species}

Given a set of species, $S$, occupying a map of sites, $I$, in different time periods, $T$, the selection of a CCC, $c \in C$, for a species, $s$, is controlled by a binary variable $\left(z_{s}^{c}\right)$ such that $z_{s}^{c}=1$ if $c$ is selected for $s$ (i.e. included in an independent subset of CCCs for species $s$ ) and $z_{s}^{c}=0$, otherwise. The variables $x_{i}^{t}$ indicate if a site $i$ is part of at least one selected CCC at time period $t$. We denote the set of all CCCs by $C$ and $C^{i t}$, the subset of CCCs in $C$ that include site $i$ in time period $t$. The following set of constraints is common to the three problems presented below:

$$
\left\{\begin{array}{lc}
\sum_{c \in C^{i t}} z_{s}^{c} \leq 1, & \forall s \in S \wedge \forall i \in I \wedge \forall t \in T \\
x^{i t} \geq z_{s}^{c}, & \forall s \in S \wedge \forall i \in I \wedge \forall t \in T \wedge \forall c \in C^{i t} \\
z_{s}^{c} \in\{0,1\}, & \forall s \in S \wedge \forall c \in C \\
x^{i t} \in[0,1], & \forall s \in S \wedge \forall c \in C
\end{array}\right.
$$

The inequalities (1) imply that, among the CCCs from $C$ that use site $i$ at time period $t, C^{i t}$, only one is considered for species $s$ (i.e. only independent corridors are accounted for each species s). Inequalities (2) relate 
the selection of a CCC, $c$, for some species with the selection of the sites included in $c$. If $c$ is selected (i.e. $z_{s}^{c}=1$ ) and includes the site $i$ at time period $t$, then $i$ will be selected for time period $t$ (i.e. $x^{i t}=1$ ). Specifically, if $z_{s}^{c}=1$ then $x^{i t}=1$ and if $z_{s}^{c}=0$ then $x^{i t} \geq 0$. Since the problems will favour solutions with the least number of sites (see below) in the final solution, $x^{i t} \geq 0$ will turn to $x^{i t}=0$. Equations (3) and (4) define the domain of the variables: $z_{s}^{c}$ are binary while $x^{i t}$, although not explicitly defined as binary, will only take zero-or-one values in the final solutions.

Furthermore, all problems share the following constraints:

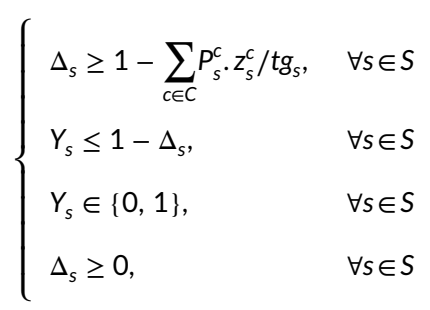

Variable $Y_{s}$ indicates whether species $s$ meets target $\operatorname{tg}_{S}\left(Y_{s}=1\right)$ or not $\left(Y_{s}=0\right)$. When $\operatorname{tg}_{s}$ is missed, that is, $\sum_{c \in C} P_{s}^{c} . z_{s}^{c}<\operatorname{tg}_{s}$, the lower bound of target shortfall, $\Delta_{s}$ is positive, then inequalities (6) force $Y_{s}<1$, and from constraints (7), $Y_{s}=0$ must hold. When no shortfall exists, the righthand side of inequalities (5) is less than or equal to zero. Constraints (6-8) allow $Y_{s}=1$, but since the problems favour species targets to be fulfilled (see constraints 10 and 11), whenever possible $Y_{s}=1$ will hold.

Here, we opt to define shortfalls as fractional values of the established targets $\sum_{c \in C} P_{s}^{c} \cdot z_{s}^{c} / \operatorname{tg}_{s}$ (in inequalities (5)). This should be the favoured approach when persistence targets for the concerning species vary by several orders of magnitude. When this is not the case, then an absolute target shortfall may be used. In this case inequalities (5) are $\Delta_{s} \geq \operatorname{tg}_{s}-\sum_{c \in C} P_{s}^{c} \cdot z_{s}^{c}$, and constraints (9) substituted with $Y_{s} \leq 1-\Delta_{s} / \operatorname{tg}_{s}$.

The problems formulated here vary in their main goals and in a small set of constraints.

\section{3 | The K-minCost problem}

We start by formulating the climate change version of minCost ( $K$-minCost) using CCCs as planning units. In this problem, the goal is the selection of a set of CCCs of minimum total cost (or area) that fulfils the established persistence targets:

$$
\min \sum_{t \in T} \sum_{i \in l} \operatorname{cost}^{i t} \cdot x^{i t}
$$

with constraints (1-8), and a lower bound on the number of species to be adequately covered:

$$
\sum_{s \in S} Y_{s} \geq K
$$

which forces at least $K$ species $(K \leq|S|)$ to have their targets fulfilled in the selected CCCs. The lower the value of $K$, the more likely a feasible solution exists.

\subsection{The B-maxCoverage problem}

The B-maxCoverage expresses the maxCoverage in the context of CCCs. An optimal solution for B-maxCoverage maximizes the number of target-fulfilled species, that is,

$$
\max \sum_{s \in S} Y_{s}
$$

Constraints (1-4) assign sites to the CCCs in solution and constraints (5-8) control target shortfalls and species statuses (i.e. target-fulfilled or shortfall species). The problem also includes budget constraints expressed by inequalities (12) or (13):

$$
\begin{gathered}
\sum_{t \in T} \sum_{i \in l} \operatorname{cost}^{i t} \cdot x^{i t} \leq B, \\
\sum_{i \in l} \operatorname{cost}^{i t} \cdot x^{i t} \leq B^{t}, \quad \forall t \in T,
\end{gathered}
$$

where $\operatorname{cost}^{i t}$ indicates the cost (or area) associated with site $i$ at time period $t$. Given that $x^{i t}<1$ and $x^{i t}=1$ when at least one CCC including site $i$ at time $t$ is selected, then the cost of site $i$ at time $t$ is counted at most once (cost ${ }^{i t}$ ).

Inequalities (12) or (13) are used depending whether the budget $(B)$ is for the whole time horizon or the budget $\left(B^{t}\right)$ is to be used for each time period $(t)$ separately.

\section{5 | The BK-minShortfall problem}

We also formulate a novel problem (BK-minShortfall), which combines the two previous problems to search for PA solutions in which the sum of target shortfalls for all species is minimized:

$$
\min \sum_{s \in S} \Delta_{s}
$$

subject to constraints (1-8), (10) and (12) or (13).

Contrary to $K$-minCost, in BK-minShortfall, $K=0$ is a plausible choice, should planners consider a solution satisfactory when the targets of all species are missed but by the smallest amount (see Table 2 for a general description and relationships between the three PA selection problems).

\section{6 | Case study data}

We use a set of 10 mammal species of conservation concern in Iberia (Table 3) to illustrate and compare the use of the three PA selection problems. These are species that (a) are listed in the Portuguese and Spanish Red List Books (Cabral et al., 2005; Dirección general para la biodiversidad, 2007) and (b) are likely to be negatively impacted by climate change in the region, both inside and outside PAs (Araújo et al., 2011). 
TABLE 2 A summary of the CCC selection problems, including the objective function, the species scope and the major constraint types (numbers in parentheses refer to the respective formulas in the Section 2)

\begin{tabular}{|c|c|c|c|c|}
\hline & maxPers & $K-\min$ Cost & B-maxCoverage & BK-minShortfall \\
\hline Scope & Single species & \multirow{2}{*}{\multicolumn{3}{|c|}{ Multiple species }} \\
\hline \multirow[t]{3}{*}{ Constraints } & $\begin{array}{l}\text { Independent } \\
\text { corridors } \\
(1-4)\end{array}$ & & & \\
\hline & - & \multicolumn{3}{|c|}{$\begin{array}{l}\text { Identify target-fulfilled species and for the remaining quantify target shortfall } \\
(5-8) \text { - }\end{array}$} \\
\hline & - & $\begin{array}{l}\text { Number of target-fulfilled } \\
\text { species, } K \\
\text { (10) }\end{array}$ & - & $\begin{array}{l}\text { Number of target-fulfilled } \\
\text { species, } K \\
\text { (10) }\end{array}$ \\
\hline
\end{tabular}

TABLE 3 List of the species considered and indices defining their distribution in lberia. Extended number of CCCs: number of usable CCCs after defining the 500 (or the maximum number possible) CCCs where each species presents the largest persistence scores; Total climatic suitability at baseline period and 2080 within the largest-persistence CCCs; Maximum persistence: The maximum persistence of a species assuming a stable current climate in the future; Persistence target: the target used within the CCC selection problems

\begin{tabular}{|c|c|c|c|c|c|c|c|c|c|}
\hline \multirow[b]{2}{*}{ Species name } & \multirow[b]{2}{*}{ Abbreviation } & \multicolumn{2}{|c|}{ Red list status } & \multirow{2}{*}{$\begin{array}{l}\text { Maximum } \\
\text { dispersal rate } \\
\text { (km/30 year) }\end{array}$} & \multirow{2}{*}{$\begin{array}{l}\text { Extended } \\
\text { number of } \\
\mathrm{CCCs}\end{array}$} & \multicolumn{2}{|c|}{$\begin{array}{l}\text { Total suitability in } \\
\text { CCCs }\end{array}$} & \multirow{2}{*}{$\begin{array}{l}\text { Maximum } \\
\text { persistence }\end{array}$} & \multirow{2}{*}{$\begin{array}{l}\text { Persistence } \\
\text { target }\end{array}$} \\
\hline & & Portugal & Spain & & & Baseline & 2080 & & \\
\hline Galemys pyrenaicus & Gpy & VU & VU & 20 & 1,473 & 306.83 & 154.78 & 4.07 & 2.40 \\
\hline Mustela erminea & Mer & DD & DD & 10 & 390 & 40.68 & 62.29 & 1.86 & 1.62 \\
\hline Mustela lutreola & Mlu & NT & EN & 10 & 171 & 7.52 & 12.24 & 0.03 & 0.03 \\
\hline Arvicola sapidus & Asa & NT & VU & 31 & 2,476 & 365.78 & 297.72 & 34.60 & 3.30 \\
\hline Microtus cabrerae & Mca & VU & VU & 115 & 811 & 227.18 & 97.32 & 0.21 & 0.21 \\
\hline Canis lupus & $\mathrm{Clu}$ & EN & NT & 160 & 2,372 & 331.21 & 360.40 & 3.76 & 2.32 \\
\hline Ursus arctos & Uar & EX & $\mathrm{CR}$ & 200 & 892 & 144.99 & 198.34 & 0.08 & 0.08 \\
\hline
\end{tabular}

Abbreviations: CE, critically endangered; DD, data deficient; EN, endangered; EX, extinct; NT, near threatened; VU, vulnerable.

${ }^{a}$ Endemic of Iberian Peninsula.

We used a set of habitat suitability models to predict changes in local climatic suitability for each species in each of 2,310 (10 arc$\min \times 10$ arcmin) sites in four time periods, from a baseline period up to 2080, in Iberia (for a comprehensive description see Araújo et al., 2011 and Supporting Information S1, Section A), assuming a plausible scenario of climate change: A1F1 (see IPCC, 2007). For each site, we also inferred a proxy of protection cost changing over time and dependent on the changing land use coverage (see Supporting Information S1, Section B). We make some simplistic assumptions to characterize species' dispersal. Specifically, we considered that dispersal success (a) depends only on geographical distances between source and settlement areas; (b) does not depend on the suitability of both those areas and (c) does not change with time. Species dispersal parameterization and protection cost of each site in each time period $\left(\operatorname{cost}^{i t}\right)$ follows data in Alagador and Cerdeira (2018; Figure 1b).

There are as many as $3 \times 10^{13} \mathrm{CCCs}$, but species persistence along the great majority of these CCCs is equal to or very close to zero (Figure S2 in Supporting Information S2). To cope with the large number of CCCs, we followed the procedure suggested above: for each species s, we ran the $k$-shortest path algorithm of Martins et al. (1999) to generate a candidate set of the $n_{s}$ largest persistence CCCs. Although many of our studied species had thousands of non-zero CCCs, in this illustrative example we selected $n_{s}=500$, because it seemed to provide enough independent CCCs to ensure that optimal or near optimal solutions were not missed. For the two species 
(a)

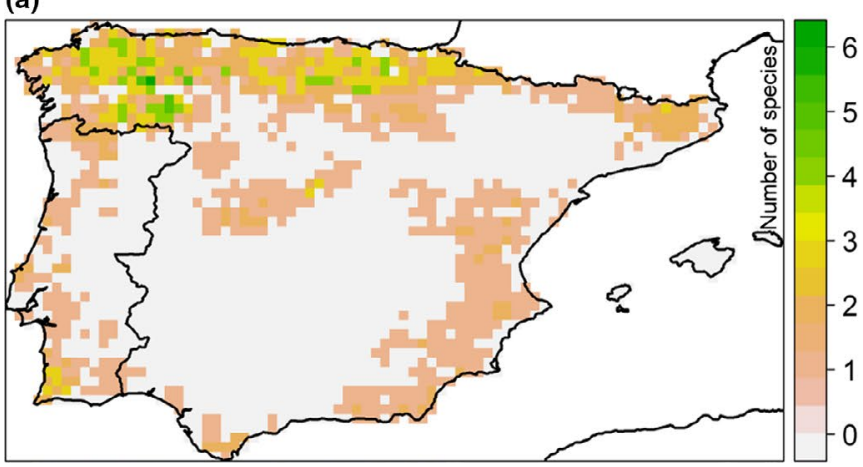

(b)

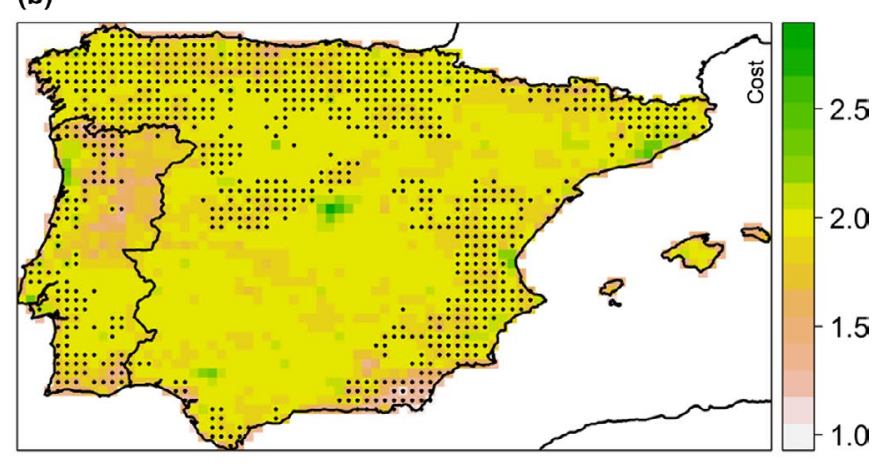

FIGURE 1 Maps characterizing the analysed region (Iberian Peninsula). (a) Number of species for which each site enters a CCC. (b) Conservation cost associated with each site. Dots refer to the sites that define CCCs for at least one species

which had fewer than 500 CCCs with a non-zero probability of persistence, we let $n_{s}$ to be equal to the number of these CCCs (Figure S2 in Supporting Information S2).

To establish persistence targets for species, we proceeded as follows. We first obtained what we call reference persistence targets, which are maximum persistence along independent CCCs under an ideal scenario of unlimited budget and with a stable climate up to 2080 (Figure 1a). This corresponds, for each species $s$, to the optimal value of the maxPers problem obtained with:

$$
\max \sum_{c \in C} P_{s}^{c} \cdot z_{s}^{c}
$$

subject to constraints (1-4).

These reference targets maxPers $s_{s}$ were then rescaled $\left(\operatorname{tg}_{s}\right)$ such that the species with lowest persistence expectancies were prioritized over the species deemed 'more persistent' (see Supporting Information S1, Section C). In order to test responses to distinct target sizes, we defined two target settings: a more demanding setting, where $\operatorname{tg}_{s}$ were directly taken as the persistence targets, and a less demanding persistence setting where we let $\operatorname{tg}_{S}=\operatorname{tg}_{s} / 2$ (see Supporting Information S1, Section C).

The K-minCost, B-maxCoverage, and KB-minShortfall problems were run for the 10 species and a range of different budgets using the IBM ILOG CPLEX callable library for C programming (IBM, 2013), with optimality gap equal to $1.0 \%$. The persistence for each species and costs associated with the CCCs identified in final solutions were recorded (see Supporting Information S1, Section D). Alternative solvers (e.g. freely available software such as SYMPHONY) can be used. We provide users a novel area selection software for depicting the "top-persistence" CCCs for each single species and for solving the three problems (see Data availability statement).

\section{\begin{tabular}{l|l}
3 & RESULTS
\end{tabular}}

The persistence scores associated to the top-persistence CCCs, optimally obtained for each single species, were quite low even under the referential target. Values ranged from 0.03 for Mustela lutreola to 88.04 for Oryctolagus cuniculus (Table 3 ), against the maximum potential expected persistence score of 500 expected surviving populations by 2080 .

Results revealed that increasing $K$ and $B$ in $K$-minCost and $B$-maxCoverage resulted in either similar or differentiated responses for both problems with regard to solution costs and target-fulfilled species. In $K$-minCost, increasing $K$ led to an exponential increase of total solution cost in both target settings, with the solutions obtained for the less demanding targets, in general, two to four times cheaper than $K$ equivalent solutions for the more demanding targets (Figure 2d). Importantly, when using the more demanding persistence targets, no more than six species $(K=6)$ had their targets fulfilled with $K$-minCost problem (Figure $2 a$ ).

Among runs of $\mathrm{K}$-minCost, each unitary increment in $\mathrm{K}$ resulted in a new target-fulfilled species joining a stable set of target-fulfilled species obtained with lower $K$ values (Figure $3 a$ ). It should be clear that this nested pattern, attained with the instances used here, is not a typical feature of the problem, and was not obtained with $B$ maxCoverage, in which increases in $B$ produced replacements among species (i.e. turnover among target-fulfilled species; Figure 3b; Figure S4 in Supporting Information S2). For each of the target settings, the species with the lowest representation were the same in $\mathrm{K}$-minCost and B-maxCoverage. With the more demanding targets, Galemys pyrenaicus, Mustela erminea, M. lutreola and Ursus arctus showed shortfalls larger than $75 \%$ of their established persistence targets (Figure 3a). With the less demanding target, G. pyrenaicus, M. Iutreola, Capra pyrenaica and Microtus cabrerae presented the largest shortfalls for most of the tested runs and their targets were the last to be met, when $K$ and $B$ increased (Figure S4 in Supporting Information S2).

With the BK-minShortfall framework, the total target shortfall decreased exponentially with a linear increase of budget, regardless of $K$ (Figure $2 c$ ). For both target scenarios, once an increase in budget allowed a new species to meet its target, no substantial change in the total target shortfall was observed thereafter. However, under the more demanding targets and $K \in\{5,6\}$, the extra target-fulfilled species resulted in a small increase of the total target shortfall in the solution (Figure 2c), which (negatively) affected G. pyrenaicus, 
FIGURE 2 Performance of solutions obtained with the three area selection problems under different parameterization regimes. Analyses with $\mathrm{K}$-minCost were performed with variation of the minimum number of species with persistence targets fulfilled, (i.e. targetfulfilled species, $K$ ); B-maxCoverage analyses were undertaken with variation of the budget available $(B)$ to conserve the selected areas over time (i.e. CCCs); BKminShortfall analyses were conducted for all using the ratio of the sum of species' persistence scores in solutions to the sum of persistence targets. Plots refer to the more (upper row) and the less (lower row) demanding persistence targets assessed

FIGURE 3 Persistence target shortfalls (as percentage of targets) obtained for each species in solutions of varying cost (budget) obtained using: (a) K-minCost, (b) B-maxCoverage and; (c) BK-minShortfall. Plots refer to the more demanding persistence targets assessed. Gpy, Galemys pyrenaicus; Mer, Mustela erminea; Mlu, Mustela lutreola; Ocu, Oryctolagus cuniculus; Asa, Arvicola sapidus; Mca, Microtus cabrerae; Clu, Canis lupus; Uar, Ursus arctos; Fsy, Felis sylvestris; Cpy, Capra pyrenaica
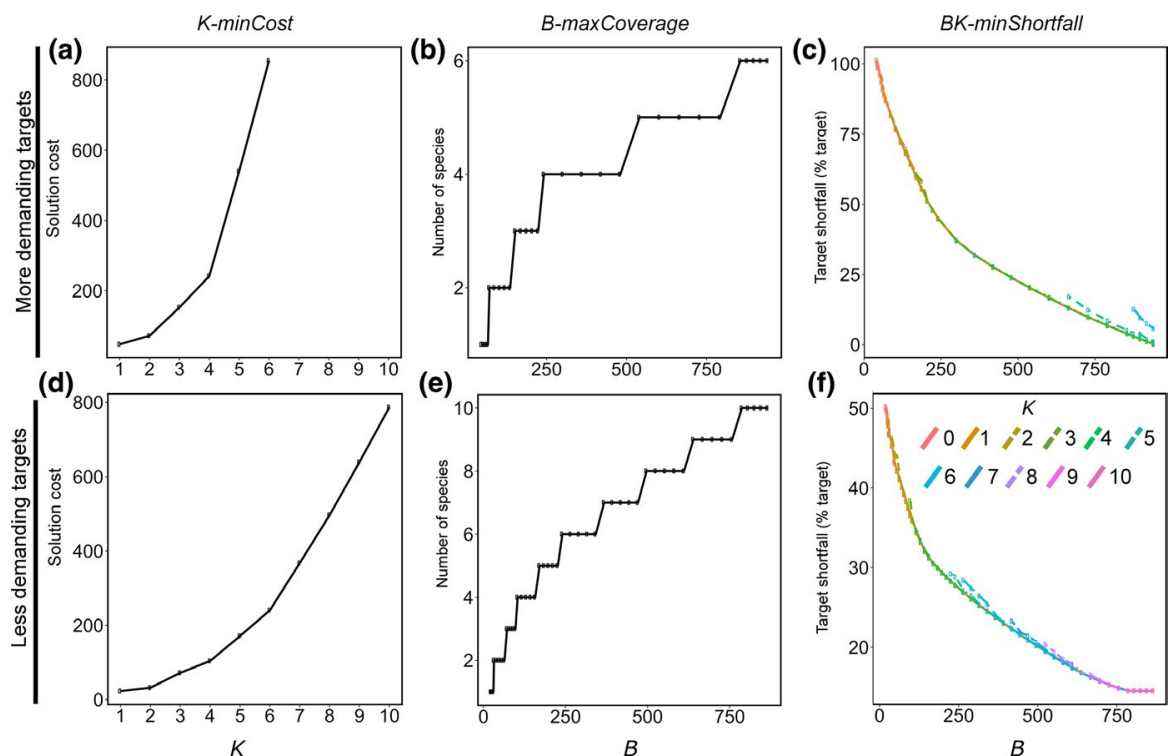

(a) K-mincost

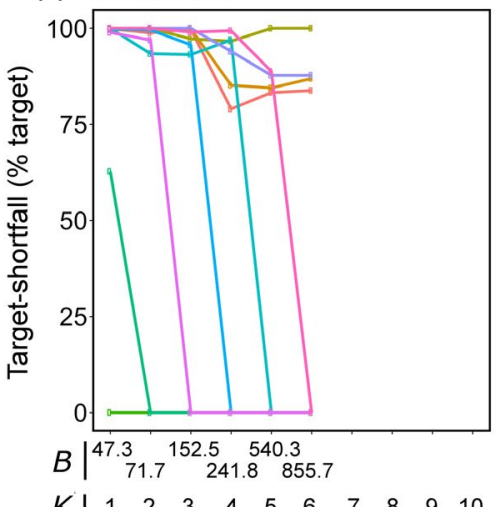

(b) B-maxCoverage

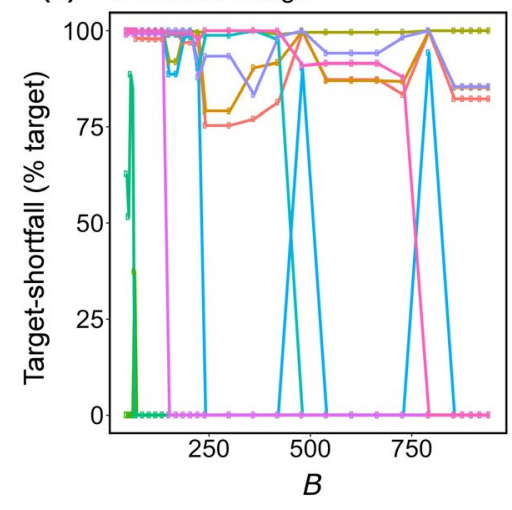

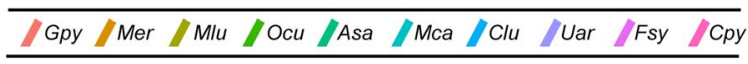

(c) BK-minShortfall

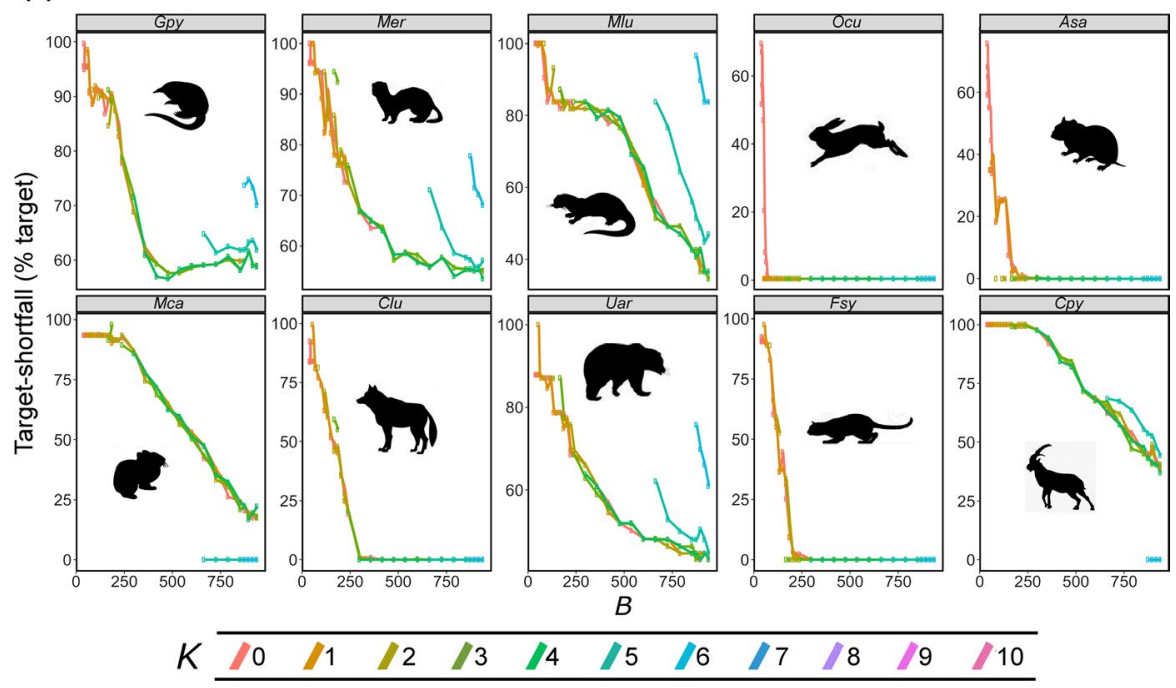

M. erminea, M. lutreola and $U$. arctus (Figure 3c). These were the species that had the poorest representation under K-minCost and BmaxCoverage (see above). For the distinct species, target shortfalls varied differently with budget increment (regardless of $K$ ). Under both target settings, four species were adequately protected with the lowest budgets tested (i.e. first quartile; O. cuniculus, Arvicola sapidus, Canis lupus and Felis sylvestris). For the remaining species, concave (G. pyrenaicus, M. erminea and U. arctus, in the more 
demanding target scenario), linear ( $M$. cabrerae and C. pyrenaica in both scenarios) and zigzagging response curves were observed (Figure 3; Figure S4 in Supporting Information S2).

The relative costs of the corridors selected to protect each species (i.e. the fraction of the total solution cost assigned for the conservation of CCCs for a given species; see Supporting Information S1, Section E) were approximately maintained (i.e. have similar rank) among the different problems and target scenarios (Figure S5 in Supporting Information S2). As expected, the species with the largest target shortfalls (M. lutreola) absorbed the lowest financial resources. In contrast, CCCs for O. cuniculus and $A$. sapidus were the most expensive, requiring on average $60 \%-75 \%$ of the total investments, among the tested $K$ and/or $B$. The cost-effectiveness (i.e. target shortfall by unit cost) related to each species presented much more variation among problems (especially between $\mathrm{K}$-minCost and B-maxCoverage) and target scenarios (Figure S6 in Supporting Information S2). In general, species reached the highest scores in $\mathrm{K}$-minCost solutions obtained with the more demanding targets. Solutions for O. cuniculus, A. sapidus and $U$. arctus were more cost-effective when compared with solutions for the remaining species. Contrastingly, G. pyrenaicus obtained the smallest benefits for each unit of investment made in the allocation of PAs.

The locations of the CCCs selected for each species and their functionality (i.e. climatic suitability associated to their sites; Figure 4; Figures S7 and S9 in Supporting Information S2) displayed a significant but weak relationship with the locations and functionality of the CCCs available for selection in Iberia, $C\left(R^{2}<0.311, p<0.005\right.$; Table S1 in Supporting Information S3). However, as expected, in each of the target settings and PA selection problem, the similarities between the CCCs in solutions and the CCCs in the selectable pool of $n_{s}$ CCCs increased with decreasing constraint tightness. Although non-linear, this trend was more evident among the CCC functionality comparisons.

In general, the sets of factors most related to species target fulfilment were different for each distinct species, varying with PA selection problems and target settings (Figure 5; Figure S8 in Supporting Information S2). Budget was the factor most prevalent in the top three predictor sets and, as expected, always with a negative sign (i.e. the larger the budget the smaller the target shortfalls). Oryctolagus cuniculus, G. pyrenaicus and M. erminea were the species more frequently selected as important predictors of species target
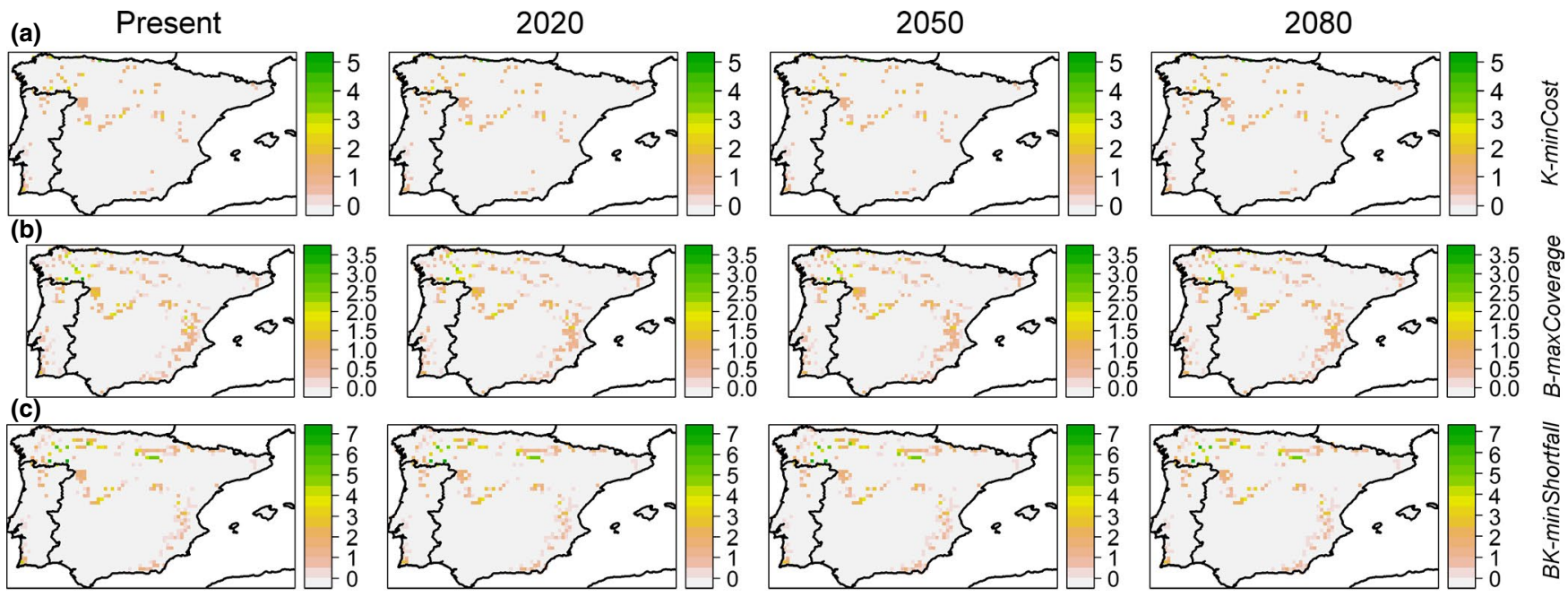

(d) K-minCost
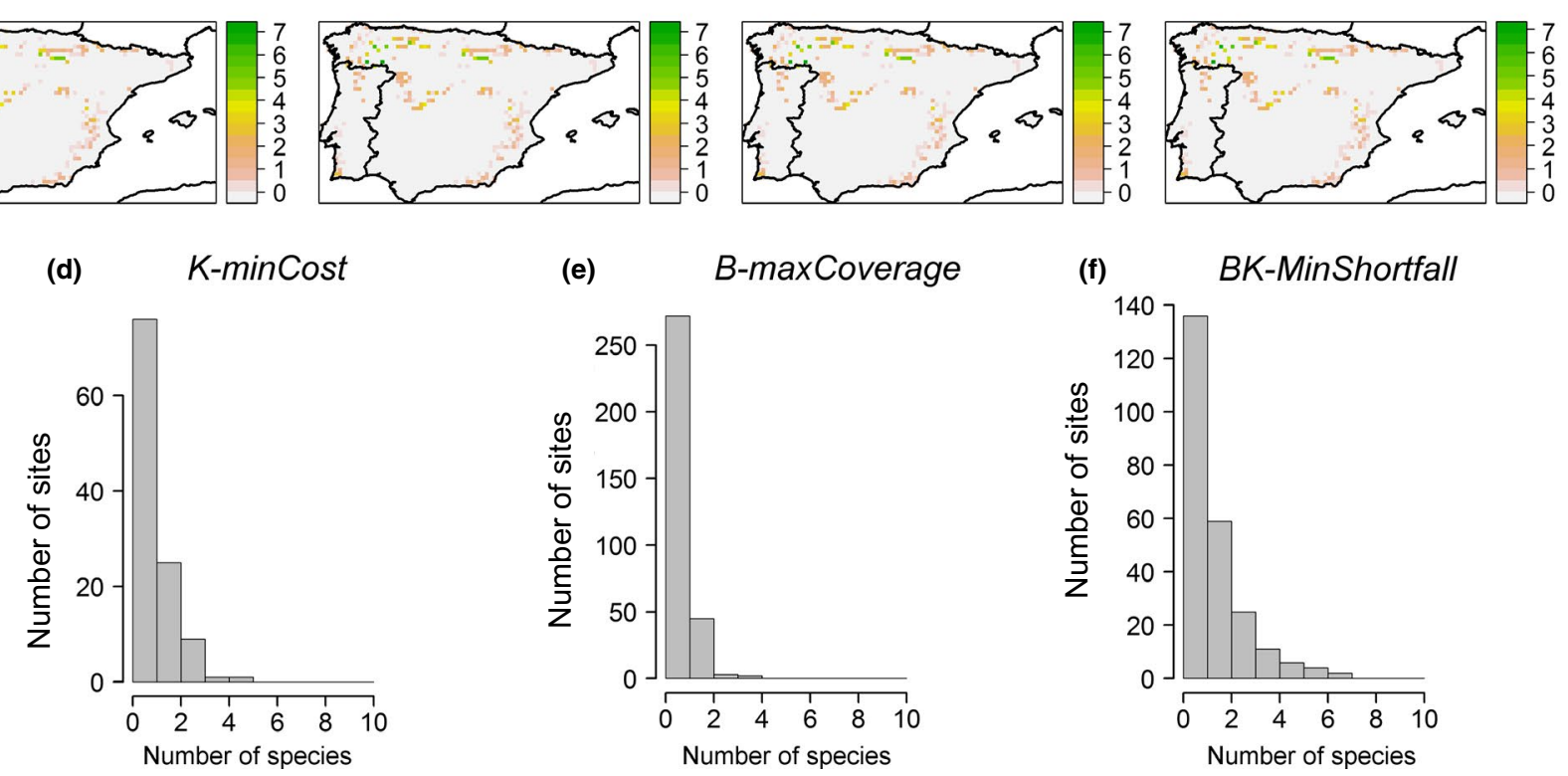

FIGURE 4 Maps summarizing (a) K-minCost, (b) B-maxCoverage and (c) BK-minShortfall solutions obtained for a number of runs with varying parameterization. The coloured sites are scored according to the average number of species that required those sites among the tested runs. The histograms present the distribution of non-null average species number in maps (d) K-minCost, (e) B-maxCoverage and (f) BKminShortfall. The figure refers to the more demanding persistence targets assessed 
FIGURE 5 Number of times (among nine tests) that each species, and budget, occurred as one of the three factors that most limit the fulfilment of persistence targets of the remaining species. (a) solutions from the K-minCost problem, (b) solutions from the B-maxCoverage problem and (c) solutions from the BK-minShortfall problem. Table in (d) details the information of pie charts by distributing counts among limiting ranks (i.e. P1, P2, P3 refer to the first, second and third most limiting factors respectively). The figure refers to the more demanding persistence targets assessed. Gpy, Galemys pyrenaicus; Mer, Mustela erminea; Mlu, Mustela lutreola; Ocu, Oryctolagus cuniculus; Asa, Arvicola sapidus; Mca, Microtus cabrerae; Clu, Canis lupus; Uar, Ursus arctos; Fsy, Felis sylvestris; Cpy, Capra pyrenaica; BGT, budget (a) K-minCost

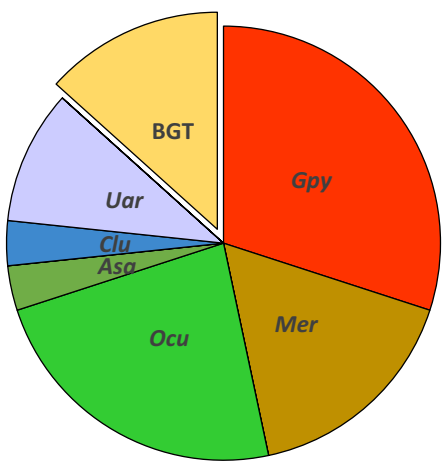

(c) BK-minShortfall

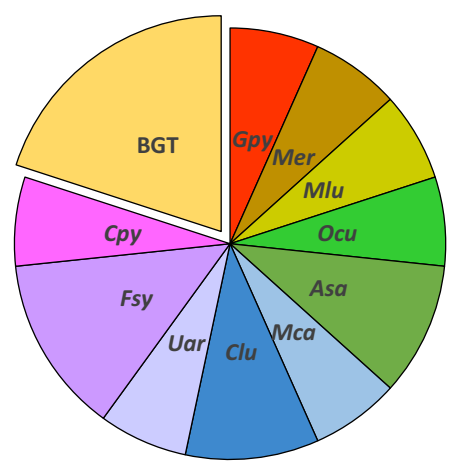

(b) B-maxCoverage

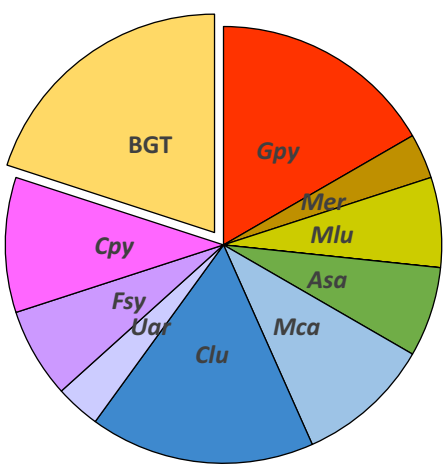

(d) Top3 limiting factors

Gpy Mer Mlu Ocu Asa Mca Clu Uar Fsy Cpy BGT K-minCost

\begin{tabular}{llllllllllll}
\hline P1 & 2 & 0 & 0 & 7 & 0 & 0 & 0 & 1 & 0 & 0 & 0 \\
P2 & 6 & 2 & 0 & 0 & 0 & 0 & 1 & 0 & 0 & 0 & 1
\end{tabular}

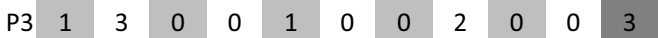
B-maxCoverage

\begin{tabular}{lllllllllllll}
\hline P1 & 5 & 0 & 0 & 0 & 1 & 1 & 1 & 1 & 1 & 0 & 0 \\
P2 & 0 & 1 & 1 & 0 & 1 & 0 & 4 & 0 & 1 & 2 & 0 \\
P3 & 0 & 0 & 1 & 0 & 0 & 2 & 0 & 0 & 0 & 1 & 6
\end{tabular}

BK-minShortfall

\begin{tabular}{lllllllllllll}
\hline P1 & 2 & 2 & 0 & 1 & 1 & 1 & 1 & 2 & 0 & 0 & 0 \\
P2 & 0 & 0 & 1 & 0 & 2 & 1 & 2 & 0 & 3 & 1 & 0 \\
P3 & 0 & 0 & 1 & 1 & 0 & 0 & 0 & 0 & 1 & 1 & 6
\end{tabular}

fulfilment (especially with $\mathrm{K}$-minCost), exhibiting strong positive and negative relationships. For B-maxCoverage and BK-minShortfall, there were several different species whose protection most limited the protection of the remaining species (Tables S2a and S2b in Supporting Information S3).

\section{4 | DISCUSSION}

Here, we addressed three distinct (but related) problems concerning the scheduling of PAs to optimize the persistence of species (or other biological features) under dynamic environments (e.g. climate change) and budgetary constraints. We extended two classical problems in reserve design and introduced a third one that runs with a min-shortfall objective function. These problems work with CCCs as planning units, in which persistence is quantified and used to set conservation targets. As noted above (in Section 2), the computational burden of handling too many CCCs may be a serious problem, and we suggested considering, for each species, a subset of the $n_{s}$ largest persistence CCCs. Finding this subset can be efficiently achieved by solving the $k$-shortest path problem, with $k=n_{s}$. Adequate subsets of CCCs, that is, that include independent CCCs on which species persistence met the conservation targets that were established for species $s$, can be determined solving the $k$-shortest path problem for different values of $k=n_{s}$, for example using a binary search algorithm. It may be advisable to enlarge the set of CCCs with some low cost CCCs, provided that their persistence for species is not insignificant, to avoid missing optimal or near optimal prioritizations.

The minCost and $\mathrm{K}$-minCost problems are better for finding solutions in which the established targets for all, and for $K$, species, respectively, are fulfilled with the minimum cost. The definition of realistic conservation targets is a key stage in the conservation planning process that should be carefully achieved, based on the most reliable data available. However, some (ideal) referential targets may be impossible to fulfil, especially when CCC sets of high persistence scores are highly different among species (i.e. no common sites). Depending on the available budgets, planners may consider certain levels of target shortfall acceptable. Several proposals have been published in this regard. For example, in Marxan (Ball et al., 2009), the fulfilment of conservation targets is not a requirement of final solutions (i.e. targets are integrated as parcels in the objective function) and weights enable species to be prioritized differently. Also, in Marxan, Game, McDonald-Madden, Puotinen, and Possingham (2008) and Game, Watts, Wooldridge, and Possingham (2008) introduced a probabilistic representation of the likelihood of targets being fulfilled, based on climate change risk assessments. The balance between target sizes and their probabilities of fulfilment allows species' weights to be defined accordingly and, consequently, the relative influence of species on the solution can be controlled. Here, we propose a different approach towards target shortfalls. Planners may decide to withdraw the shortfall species from the original planning design (after a K-minCost problem initially infeasible was turned solvable with a lower $K$ value), thus leading to savings of financial 
resources (in our analyses solution costs were exponentially related to $K$, Figure 2). The shortfall species may then be managed with alternative strategies to PAs, for example, through ex-situ (e.g. assisted colonization) or habitat restoration actions that in the ultimate case may promote the natural re-adaptation of species ranges to climate change in the new colonized or restored areas (Shoo et al., 2013; Thomas et al., 2011). Still, when using the $K$ parameter, planners may also consider some particular species, $\tilde{s}$, of high priority to protect. These cases can be easily integrated into the problems proposed here by adding an additional constraint to the formulation to force the inclusion of that species $\left(Y_{\tilde{s}}=1\right)$. However, when doing this, problems may become infeasible if the targets for these species are impossible to meet, even if all sites in the conservation planning scenario are protected and so conservation planners will need to lower the persistence targets.

The B-maxCoverage problem reflects the typical context of real-world conservation plans, in which scarce financial resources severely constrain conservation effectiveness (Brown et al., 2015). Since the problem is built around a fixed budget, solutions tend to be financially suboptimal (i.e. solutions of similar ecological effectiveness may be achieved with less financial effort, see the step-like responses in Figure $2 \mathrm{~b}, \mathrm{e})$. Planners may use this feature of B-maxCoverage, combined with the K-minCost problem, to minimize solution cost with the maximum levels of target achievement. First, the maximum number of target-fulfilled species $\left(k^{B}\right)$ can be obtained from $B$-maxCoverage using the budget available $(B)$; next the $K$-minCost is run with $K=k^{B}$, and letting $b^{K}$ be the resulting cost. Planners have the guarantee that at least $k^{B}$ species are entirely target-fulfilled with a budget $b^{K} \leq B$ (i.e. saving $B-b^{K}$ ). Clearly, this may also be performed in reverse order (i.e. solving $K$-minCost first and then $B$-maxCoverage) to determine the minimum budget $b^{K}$ that ensures a given number $K$ of species to be target-fulfilled, and then finding whether more than $K$ of species can be target-fulfilled with budget $b^{K}$.

The BK-minShortfall problem integrates the target constrains of $K$-minCost with the budgetary constraint(s) of B-maxCoverage. In this new problem, feasibility (i.e. a solution that covers all constraints) is balanced with solution quality (i.e. effectiveness, measured by the number of target-fulfilled species and by the number of target shortfalls) by varying $B, K$ (or both) values. Using this problem, planners can generate a portfolio of solutions based on different budgets and target-fulfilled species, with the aim of conducting sensitivity analyses relating resources available and target fulfilment, while maximizing the persistence of the shortfall species.

Here, we assessed persistence of species using a simple measure based on two elementary autoecological principles considered significant at large biogeographic scales (Phillips et al., 2008): the suitability of each site to sustain viable populations of a species within a given time period; and the ability of species to respond to local climatic disturbances through dispersal and range readjustments. Depending on the spatial, temporal and taxonomic scales and the corresponding levels of accuracy required, data can be refined, and their interdependencies integrated in order to estimate persistence more accurately. For example, in species exhibiting a meta-population structure, both the quality (i.e. suitability) and the connectivity (i.e. functional distance) of population cores are important factors to infer persistence of the whole meta-population (Che-Castaldo \& Neel, 2016; Christopher \& Lisa, 2009; Ovaskainen \& Hanski, 2003b; Zamborain-Mason, Russ, Abesamis, Bucol, \& Connolly, 2017). Moreover, in such meta-population models, dispersal fluxes are assumed dependent on the habitat characteristics of the source and destination areas (Hanski \& Ovaskainen, 2000). Because of the large analytical detail required to make such accurate assessments and since the purpose of our study is mostly conceptual, we chose not to undertake such assessments in our study. Other simplistic assumptions were made herein: (a) each site is large enough to support a viable population of individuals of each species in each time step. Alternatively, we also may assume a sufficient proportion of each selected site is protected; (b) independent CCCs, that is, those that do not converge in a site in a given time period, are assumed to be functionally independent. This is a strong assumption as spatial proximity and edge effects may lead interacting processes to occur between CCCs. Although mathematically possible, the control of the cohesiveness of CCCs brings still larger complexity to the problem; (c) there are no beneficial or detrimental interactions among species (most conservation planning assumes this); (d) there are no lag effects between selection of a site and its potential benefit to each species, and, related to this, (e) conservation actions to be made in a site do not change the climatic suitability for the species of concern, finally, (f) predictive uncertainty was not integrated for robust decisions to be delivered. Several methods have been proposed on how to work uncertainty into conservation planning (Regan et al., 2005). The use of quantified uncertainty data as a local cost (Lemes \& Loyola, 2013) or thorough portfolio theory (Liang et al., 2018), stochastic dynamic programming (Wilson, McBride, Bode, \& Possingham, 2006) and robust optimization (Haider, Charkhgard, \& Kwon, 2018) are examples. When uncertainty is severe and unquantifiable, then implementing info-gap theory is an effective option (Hayes, Barry, Hosack, \& Peters, 2013). All these analyses require additional computational resources and it would not be evident how these approaches could be integrated in the framework here proposed.

In general, the failure of solutions to meet (persistence) targets established for species to cope with climate change results from two factors that may act synergistically. First, targets may be missed due to ecological limitations of species. That is, species may: (a) have insufficient suitable area available within the planning region and time horizon; (b) be faced with dispersal barriers that prevent reaching suitable areas; or (c) have limited dispersal rates, that is, lower than the rate of environmental change (Fortini \& Dye, 2017). A previous study, which used minShortfall to compare the effects of climate, dispersal rates and budget (resulting from comparing single vs. multiple-species plans) on the performance of CCCs, found that budget and climate were the most limiting factors to species target fulfilment (Alagador \& Cerdeira, 2018). For only two species (C. lupus and $F$ sylvestris), dispersal ability was also a significant factor affecting conservation success either in single or multiple-species 
conservation plans. Future climate projections are expected to have a positive impact on M. erminea and U. arctus in Iberia. Therefore, the adequate representation of these species in final CCCs was uniquely dependent on the budget available to conserve all the 10 species using a multiple-species conservation plan.

When funding is scarce, target setting should be carefully designed, because a large persistence target for a species may lead other species to be inadequately protected. Here, we investigated these effects using the three PA selection problems proposed, which, combined, allowed us to perform trade-off analyses among species' persistence targets and the budget constraints. We observed that interdependencies between species targets were very contextual. With different problems and two target settings, different species limited the adequate protection of the remaining species (i.e. target shortfall). These results follow-up analyses made by Moilanen and Arponen (2011) using Zonation software (Moilanen, Kujala, et al., 2009) in which the overlapping patterns of species' ranges, combined with the costs to protect sites, emerged as the key factors that make target setting designs complex and idiosyncratic. More recently, Kaim, Watts, and Possingham (2017) used a shadows-pricing technique to fully optimize target setting in Marxan. We extended assessments on the effect of target setting to three PA selection problems that integrate climate change effects in a multi-period timeline. The complexities associated with the distribution of the available financial resources among species, in the simplest forms of the minCost and maxCoverage, are carried over (and even exacerbated) in similar PA selection problems that explicitly account for the dynamic effects of climate change on species' distributions and persistence.

A point that may be crucial and should be mentioned concerns the number of CCCs selected for each species. Expectedly, but depending on the targets established for the species, solutions included multiple independent corridors for each species, thus protecting multiple sites within a single period of time. However, this is not explicitly stated in the formulations, and only a single CCC can be selected for each species. This may not be a concern, especially if each site is large enough to secure multiple populations and preserve the species. Nevertheless, it may be appropriate to ensure at least a given number of independent CCCs, say $m_{s}$, for species $s$. This can be achieved adding to the formulations the inequality $\sum_{c \in C} Z_{s}^{c} \geq m_{s}$, for each concerned species $s$.

We conclude with a note on the computational practicality of the problems presented here. As noted above, generating the initial set of CCCs for each species to ensure optimal solutions is not an issue but, if very large sets of CCCs exist, it may take too long to solve the problems. The sizes of the instances we considered in the case study, about 5,000 CCCs (10 species, 500 CCCs per species), seem to be close to what is plausible to solve using methods providing optimality guarantee. This is especially evident for BK-minShortfall. (Tables S3 in Supporting Information S3 report the CPU time spent on each instance). For larger number of CCCs, increasing the optimality gap could be an option. Note, however, that in general, an extremely small fraction of the $|S|^{\{t N\}} \mathrm{CCC}$ s will provide species accountable persistence benefits. Thus, thousands of sites may generate only a few CCCs worth counting. Nevertheless, the problems are difficult to solve (NP-hard), and maxCoverage and BK-minShortfall are combinatorial optimization problems that are particularly involved.

With this study, we developed a software tool (iC5, see Data availability statement) which enables the identification of the top$\left(n_{s}\right)$ CCCs delivering the largest persistence scores for each species. The software also integrates optimal solvers for the three multiple species problems presented here. The development of heuristics to handle very large instances is a possibility that we plan to address in future work and that will be fully integrated in an upgraded version of iC5.

\section{ACKNOWLEDGEMENTS}

We thank Monica Papeş, Justin Roborg-Söndergaard, Hsiao-Hsuan Wang and four anonymous reviewers for valuable feedback. This work was funded by National funds through the Fundação para a Ciência e a Tecnologia (FCT) under the project UIDB/05183/2020 and also by FEDER funds and Programa Operacional Factores de Competitividade - COMPETE - through FCT's project PTDC/AAGGLO/3979/2014 (ref. 9471-RIDTI). DA received support through a FCT's postdoctoral fellowship SFRH/BPD/104077/2014. The authors have no conflicts of interest to declare. [Correction added on 28 August 2020, after first online publication: the acknowledgment section has been updated.]

\section{AUTHORS' CONTRIBUTIONS}

D.A. and J.O.C. jointly conceived the ideas, planned the methodology, analysed the data and wrote the paper. The authors gave final approval for publication and stated they have followed all the codes of conduct for peer-reviewed publication.

\section{PEER REVIEW}

The peer review history for this article is available at https://publo ns.com/publon/10.1111/2041-210X.13455.

\section{DATA AVAILABILITY STATEMENT}

At https://doi.org/10.5281/zenodo.3932003 readers may find a software tool (iC5: Climate Change-Concerned Conservation Corridors: an identifier) to handle and solve the problems presented here with their own data and specifications (Alagador \& Cerdeira, 2020). The software is currently limited to run with Windows OS. The authors are solely responsible for the content and functionality of these materials. Queries (other than absence of the material) should be directed to the corresponding author.

\section{ORCID}

Diogo Alagador (iD https://orcid.org/0000-0003-0710-3187

Jorge Orestes Cerdeira (iD https://orcid.org/0000-0002-3814-7660

\section{REFERENCES}

Alagador, D., \& Cerdeira, J. O. (2017). Meeting species persistence targets under climate change: A spatially explicit conservation planning model. 
Diversity and Distributions, 23, 703-713. https://doi.org/10.1111/ ddi.12562

Alagador, D., \& Cerdeira, J. O. (2018). A quantitative analysis on the effects of critical factors limiting the effectiveness of species conservation in future-time. Ecology and Evolution, 8, 3457-3467. https:// doi.org/10.1002/ece3.3788

Alagador, D., \& Cerdeira, J. O. (2020). Revisiting the minimum set cover, the maximal coverage problems and a maximum benefit area selection problem to make climate-change-concerned conservation plans effective (iC5 software, version 1.0.0). Zenodo, https://doi.org/ 10.5281/zenodo.3932003

Alagador,D.,Cerdeira,J.O.,\&Araújo,M.B.(2014).Shifting protectedareas: Scheduling spatial priorities under climate change. Journal of Applied Ecology, 51, 703-713. https://doi.org/10.1111/1365-2664.12230

Alagador, D., Cerdeira, J. O., \& Araújo, M. B. (2016). Climate change, species range shifts and dispersal corridors: An evaluation of spatial conservation models. Methods in Ecology and Evolution, 7, 853-866. https://doi.org/10.1111/2041-210X.12524

Albert Cécile, H., Rayfield, B., Dumitru, M., \& Gonzalez, A. (2017). Applying network theory to prioritize multispecies habitat networks that are robust to climate and land-use change. Conservation Biology, 31, 1383-1396. https://doi.org/10.1111/cobi.12943

Araújo, M. (2009). Climate change and spatial conservation planning. In A. Moilanen, H. Possingham, \& K. A. Wilson (Eds.), Spatial conservation prioritization: Quantitative methods and computational tools ( $\mathrm{pp}$. 172-184). Oxford, UK: Oxford University Press.

Araújo, M. B., Alagador, D., Cabeza, M., Nogués-Bravo, D., \& Thuiller W. (2011). Climate change threatens European conservation areas. Ecology Letters, 14, 484-492. https://doi.org/10.1111/j.1461-0248. 2011.01610.x

Bagchi, R., Crosby, M., Huntley, B., Hole, D. G., Butchart, S. H. M., Collingham, Y., ... Willis, S. G. (2013). Evaluating the effectiveness of conservation site networks under climate change: Accounting for uncertainty. Global Change Biology, 19, 1236-1248. https://doi. org/10.1111/gcb.12123

Baker, D. J., Hartley, A. J., Burgess, N. D., Butchart, S. H. M., Carr, J. A., Smith, R. J., ... Willis, S. G. (2015). Assessing climate change impacts for vertebrate fauna across the West African protected area network using regionally appropriate climate projections. Diversity and Distributions, 21, 991-1003. https://doi.org/10.1111/ ddi.12337

Ball, I. R., Possingham, H. P., \& Watts, M. (2009). Marxan and relatives: Software for spatial conservation prioritisation. In A. Moilanen, K. A Wilson, \& H. P. Possingham (Eds.), Spatial conservation prioritisation: Quantitative methods and computational tools (pp. 185-195). Oxford, UK: Oxford University Press.

Beier, P. (2012). Conceptualizing and designing corridors for climate change. Ecological Restoration, 30, 312-319. https://doi.org/10.3368/ er.30.4.312

Brown, C. J., Bode, M., Venter, O., Barnes, M. D., McGowan, J., Runge, C. A., ... Possingham, H. P. (2015). Effective conservation requires clear objectives and prioritizing actions, not places or species. Proceedings of the National Academy of Sciences of the United States of America, 112, E4342. https://doi.org/10.1073/pnas.1509189112

Cabral, M. J., Almeida, J., Almeida, P. R., Delliger, T., Ferrand de Almeida, N., \& Oliveira, M. E. ... Santos-Reis, M. (2005). Livro Vermelho dos Vertebrados de Portugal. Instituto da Conservação da Natureza. Lisboa (p. 659). Lisboa, Portugal: Instituto da Conservação da Natureza.

Camm, J. D., Norman, S. K., Polasky, S., \& Solow, A. R. (2002). Nature reserve site selection to maximize expected species covered. Operations Research, 50, 946-955. https://doi.org/10.1287/opre.50.6.946.351

Che-Castaldo, J. P., \& Neel, M. C. (2016). Species-level persistence probabilities for recovery and conservation status assessment. Conservation Biology, 30, 1297-1306. https://doi.org/10.1111/cobi. 12728
Christopher, P. G., \& Lisa, L. M. (2009). Utility of measuring abundance versus consistent occupancy in predicting biodiversity persistence. Conservation Biology, 23, 1260-1269. https://doi.org/10.1111/j.15231739.2009.01253.x

Church, R. L., Stoms, D. M., \& Davis, F. W. (1996). Reserve selection as a maximal covering location problem. Biological Conservation, 76, 105112. https://doi.org/10.1016/0006-3207(95)00102-6

D'Aloia, C. C., Naujokaitis-Lewis, I., Blackford, C., Chu, C., Curtis, J. M. R., Darling, E., ... Fortin, M.-J. (2019). Coupled networks of permanent protected areas and dynamic conservation areas for biodiversity conservation under climate change. Frontiers in Ecology and Evolution, 7. https://doi.org/10.3389/fevo.2019.00027

D'Amen, M., Bombi, P., Pearman, P. B., Schmatz, D. R., Zimmermann, N. E., \& Bologna, M. A. (2011). Will climate change reduce the efficacy of protected areas for amphibian conservation in Italy? Biological Conservation, 144, 989-997. https://doi.org/10.1016/j.biocon.2010. 11.004

Di Marco, M., Santini, L., Visconti, P., Mortelliti, A., Boitani, L., \& Rondinini, C. (2016). Using habitat suitability models to scale up population persistence targets. Hystrix, the Italian Journal of Mammalogy, 27(1), 1-7.

Dirección general para la biodiversidad. (2007). Atlas y libro rojo de los mamíferos terrestres de España. Madrid España: Organismo Autónomo Parques Nacionales.

Fortini, L. B., \& Dye, K. (2017). At a global scale, do climate change threatened species also face a greater number of non-climatic threats? Global Ecology and Conservation, 11, 207-212. https://doi.org/10.1016/j. gecco.2017.06.006

Fox, B. L. (1975). k-th shortest paths and applications to the probabilistic networks. ORSA/TIMS Joint National Meeting, 23, B263.

Game, E. T., McDonald-Madden, E. V. E., Puotinen, M. L., \& Possingham, H. P. (2008). Should we protect the strong or the weak? Risk, resilience, and the selection of marine protected areas. Conservation Biology, 22, 1619-1629. https://doi.org/10.1111/j.1523-1739.2008.01037.x

Game, E. T., Watts, M. E., Wooldridge, S., \& Possingham, H. P. (2008). Planning for persistence in marine reserves: A question of catastrophic importance. Ecological Applications, 18, 670-680. https:// doi.org/10.1890/07-1027.1

Haider, Z., Charkhgard, H., \& Kwon, C. (2018). A robust optimization approach for solving problems in conservation planning. Ecological Modelling, 368, 288-297. https://doi.org/10.1016/j.ecolm odel.2017.12.006

Hanski, I., \& Ovaskainen, O. (2000). The metapopulation capacity of a fragmented landscape. Nature, 404, 755-758. https://doi.org/ 10.1038/35008063

Hayes, K. R., Barry, S. C., Hosack, G. R., \& Peters, G. W. (2013). Severe uncertainty and info-gap decision theory. Methods in Ecology and Evolution, 4, 601-611. https://doi.org/10.1111/2041-210X.12046

IBM. (2013). IBM ILOG CPLEX 12.6 user manual. Retrieved from https:// www.ibm.com/support/knowledgecenter/SSSA5P_12.6.2/ilog. odms.studio.help/pdf/usrcplex.pdf

IPCC. (2007). Climate Change 2007: Synthesis report. Contribution of Working Groups I, II and III to the Fourth Assessment Report of the Intergovernmental Panel on Climate Change. Geneva, Switzerland: IPCC.

Jones, K. R., Watson, J. E. M., Possingham, H. P., \& Klein, C. J. (2016). Incorporating climate change into spatial conservation prioritisation: A review. Biological Conservation, 194, 121-130. https://doi. org/10.1016/j.biocon.2015.12.008

Kaim, A., Watts, M. E., \& Possingham, H. P. (2017). On which targets should we compromise in conservation prioritization problems? Methods in Ecology and Evolution, 8, 1858-1865. https://doi. org/10.1111/2041-210X.12812

Keith, D. A., Mahony, M., Hines, H., Elith, J., Regan, T. J., Baumgartner, J. B., ... AkÇAkaya, H. R. (2014). Detecting extinction risk from climate change by IUCN red list criteria. Conservation Biology, 28, 810-819. https://doi.org/10.1111/cobi.12234 
Lemes, P., \& Loyola, R. D. (2013). Accommodating species climate-forced dispersal and uncertainties in spatial conservation planning. PLoS ONE, 8, e54323. https://doi.org/10.1371/journal.pone.0054323

Liang, J., Gao, X., Zeng, G., Hua, S., Zhong, M., Li, X., \& Li, X. (2018). Coupling modern portfolio theory and marxan enhances the efficiency of lesser white-fronted Goose's (Anser erythropus) habitat conservation. Scientific Reports, 8, 214. https://doi.org/10.1038/ s41598-017-18594-2

Margules, C. R., \& Pressey, R. L. (2000). Systematic conservation planning. Nature, 405, 243-253. https://doi.org/10.1038/35012251

Martins, E. D. Q. V., Pascoal, M. M. B., \& Santos, J. L. E. D. (1999). Deviation algorithms for ranking shortest paths. International Journal of Foundations of Computer Science, 10, 247-261. https://doi.org/ 10.1142/S0129054199000186

McIntosh, E. J., Pressey, R. L., Lloyd, S., Smith, R., \& Grenyer, R. (2017). The impact of systematic conservation planning. Annual Review of Environment and Resources, 42, 677-697. https://doi.org/10.1146/ annurev-environ-102016-060902

McLaughlin, J. F., Hellmann, J. J., Boggs, C. L., \& Ehrlich, P. R. (2002). Climate change hastens population extinctions. Proceedings of the National Academy of Sciences of the United States of America, 99, 6070-6074. https://doi.org/10.1073/pnas.052131199

Moilanen, A., \& Arponen, A. (2011). Setting conservation targets under budgetary constraints. Biological Conservation, 144, 650-653. https://doi.org/10.1016/j.biocon.2010.09.006

Moilanen, A., Kujala, H., \& Leathwick, J. R. (2009). The zonation framework and software for conservation prioritization. In A. Moilanen, H. Possingham, K. A. Wilson (Eds.), Spatial conservation prioritization: Quantitative methods and computational tools (pp. 196-210). Oxford, UK: Oxford University Press.

Moilanen, A., Wilson, K., \& Possingham, H. (2009). Spatial conservation prioritization: Quantitative methods and computational tools. Oxford, UK: Oxford University Press.

Ovaskainen, O., \& Hanski, I. (2003a). Extinction threshold in metapopulation models. Annales Zoologici Fennici, 40, 17.

Ovaskainen, O., \& Hanski, I. (2003b). How much does an individual habitat fragment contribute to metapopulation dynamics and persistence? Theoretical Population Biology, 64, 481-495. https://doi. org/10.1016/S0040-5809(03)00102-3

Phillips, S., Williams, P., Midgley, G., \& Aaron, A. (2008). Optimizing dispersal corridors for the cape proteaceae using network flow. Ecological Applications, 18, 1200-1211. https://doi.org/10.1890/07-0507.1

Regan, H. M., Ben-Haim, Y., Langford, B., Wilson, W. G., Lundberg, P., Andelman, S. J., \& Burgman, M. A. (2005). Robust decision-making under severe uncertainty for conservation management. Ecological Applications, 15, 1471-1477. https://doi.org/10.1890/03-5419

Rutherford, M. C., Powrie, L. W., \& Schulze, R. E. (1999). Climate change in conservation areas of South Africa and its potential impact on floristic composition: A first assessment. Diversity and Distributions, 5, 253-262. https://doi.org/10.1046/j.1472-4642.1999.00061.x

Saetersdal, M., Line, J. M., \& Birks, H. J. B. (1993). How to maximize biological diversity in nature reserve selection: Vascular plants and breeding birds in deciduous woodlands, western Norway. Biological Conservation, 66, 131-138. https://doi.org/10.1016/00063207(93)90144-P

Shoo, L., Hoffmann, A., Garnett, S., Pressey, R., Williams, Y., Taylor, M., ... Williams, S. (2013). Making decisions to conserve species under climate change. Climatic Change, 119, 239-246. https://doi. org/10.1007/s10584-013-0699-2

Snyder, S. A., \& Haight, R. G. (2014). Application of the maximal covering location problem to habitat reserve site selection. International Regional Science Review, 39, 28-47. https://doi.org/10.1177/01600 17614551276

Soto, C. G. (2001). The potential impacts of global climate change on marine protected areas. Reviews in Fish Biology and Fisheries, 11, 181-195.

Thomas, C. D., Cameron, A., Green, R. E., Bakkenes, M., Beaumont, L. J., Collingham, Y. C., ... Williams, S. E. (2004). Extinction risk from climate change. Nature, 427, 145-148. https://doi.org/10.1038/natur e02121

Thomas, C. D., Hill, J. K., Anderson, B. J., Bailey, S., Beale, C. M., Bradbury, R. B., ... Yardley, T. (2011). A framework for assessing threats and benefits to species responding to climate change. Methods in Ecology and Evolution, 2, 125-142. https://doi.org/10.1111/j.2041-210X. 2010.00065.x

Walther, G.-R., Post, E., Convey, P., Menzel, A., Parmesan, C., Beebee, T. J. C., ... Bairlein, F. (2002). Ecological responses to recent climate change. Nature, 416, 389-395. https://doi.org/10.1038/416389a

Watson, J. E. M., Cross, M., Rowland, E., Joseph, L. N., Rao, M., \& Seimon, A. (2011). Planning for species conservation in a time of climate change. In J. Juan Blanco \& H. Kheradmand (Eds.), Climate Change Research and Technology for Adaptation and Mitigation (pp. 379-402). Rijeka, Croatia; Shangai, China: InTech.

Watson, J. E. M., Dudley, N., Segan, D. B., \& Hockings, M. (2014). The performance and potential of protected areas. Nature, 515, 67-73. https://doi.org/10.1038/nature13947

Williams, P., Hannah, L., Andelman, S., Midgley, G., Araujo, M., Hughes, G., ... Pearson, R. (2005). Planning for climate change: Identifying minimum-dispersal corridors for the cape proteaceae.Conservation Biology, 19, 1063-1074. https://doi.org/10.1111/j.1523-1739.2005.00080.x

Wilson, K. A., McBride, M. F., Bode, M., \& Possingham, H. P. (2006). Prioritizing global conservation efforts. Nature, 440, 337-340. https:// doi.org/10.1038/nature04366

Zamborain-Mason, J., Russ, G. R., Abesamis, R. A., Bucol, A. A., \& Connolly, S. R. (2017). Network theory and metapopulation persistence: Incorporating node self-connections. Ecology Letters, 20, 815-831. https://doi.org/10.1111/ele.12784

\section{SUPPORTING INFORMATION}

Additional supporting information may be found online in the Supporting Information section.

How to cite this article: Alagador D, Cerdeira JO. Revisiting the minimum set cover, the maximal coverage problems and a maximum benefit area selection problem to make climatechange-concerned conservation plans effective. Methods Ecol Evol. 2020;00:1-13. https://doi.org/10.1111/2041210X.13455 